\title{
Pseudo-Internuclear Ophthalmoplegia as a Sign of Overlapping Myasthenia Gravis in a Patient with 'Intractable' Hypothyroidism
}

\author{
Yuu Yamazaki ${ }^{1}$, Tomohito Sugiura ${ }^{2}$ and Katsumi Kurokawa ${ }^{3}$
}

\begin{abstract}
We describe a 52-year-old man with a history of increasing fatigability and gait disturbances that were first attributed to hypothyroidism. On examination, he had bilateral pseudo-internuclear ophthalmoplegia with weakness of adduction and abducting nystagmus. Convergence was also impaired and he showed proximal weakness of the limb. Intravenous edrophonium almost completely abolished the nystagmus and adducting muscle weakness, and improved the strength of proximal muscles groups. The clinical response to the administration of edrophonium, the presence of AChR binding antibodes and the repetitive nerve stimulation test findings indicated that the patient had pseudo-internuclear ophthalmoplegia as a manifestation of generalized myasthenia gravis. Whereas hypothyroidism was effectively controlled with levothyroxine, his subsequent response to thymectomy, prednisolone and cholinesterase inhibitors confirmed the diagnosis of generalized myasthenia gravis.
\end{abstract}

Key words: autoimmune thyroiditis, hypothyroidism, myasthenia gravis, internuclear ophthalmoplegia (INO), pseudo-INO, thymoma

(Inter Med 49: 69-72, 2010)

(DOI: 10.2169/internalmedicine.49.2348)

\section{Introduction}

Myasthenia gravis (MG) occurs in fewer than $1 \%$ of dysthyroid patients (1). MG and hypothyroidism share some features, principally weakness and fatigability of voluntary muscles (2). Thus, once a diagnosis of weakness and fatigability due to hypothyroidism is established, a diagnostic pitfall overlooking an additional muscle weakness caused by a co-occurrence of MG can occur. Here, we describe a man with a diagnosis of hypothyroidism whose characteristic eye movement lead us a suspicion of concomitant MG.

\section{Case Report}

In August 2007, a 52-year-old man presented to his local hospital with a 2-week history of fatigue and upper limb weakness. He was diagnosed as having severe hypothyroid- ism. Despite treatment with levothyroxine, his symptoms progressively worsened. He presented with diplopia, which was most marked on lateral gaze bilaterally, and he became unable to walk even short distances over the subsequent 2 weeks. For further evaluation of 'intractable' hypothyroidism, he was admitted to our hospital 12 days after the initial presentation. His past history was unremarkable and he was not taking any medication except for levothyroxine.

A physical examination revealed mild goiter. Neurologically, his oculomotor abnormalities were as follows: 1) on vertical gaze, his upward gaze was slightly restricted, while his downward gaze was normal; 2) his eye convergence was disturbed; 3) on horizontal gaze, adduction paresis and monocular nystagmus of the abducted eye were observed bilaterally (Fig. 1); 4) bilateral mild ptosis was observed; 5) Hertel exophthalmometry revealed no exophthalmos. Proximal muscle weakness involving the upper and lower extremities was noted, and reflexes were diminished. Labora-

${ }^{1}$ Department of Clinical Neuroscience and Therapeutics, Hiroshima University, Hiroshima, ${ }^{2}$ Department of Neurology, National Hospital Organization Kure Medical Center and Chugoku Cancer Center, Kure and ${ }^{3}$ Department of Neurology, Kawasaki Medical School, Kurashiki Received for publication April 8, 2009; Accepted for publication September 9, 2009

Correspondence to Dr. Yuu Yamazaki, yuu.yamazaki@gmail.com 

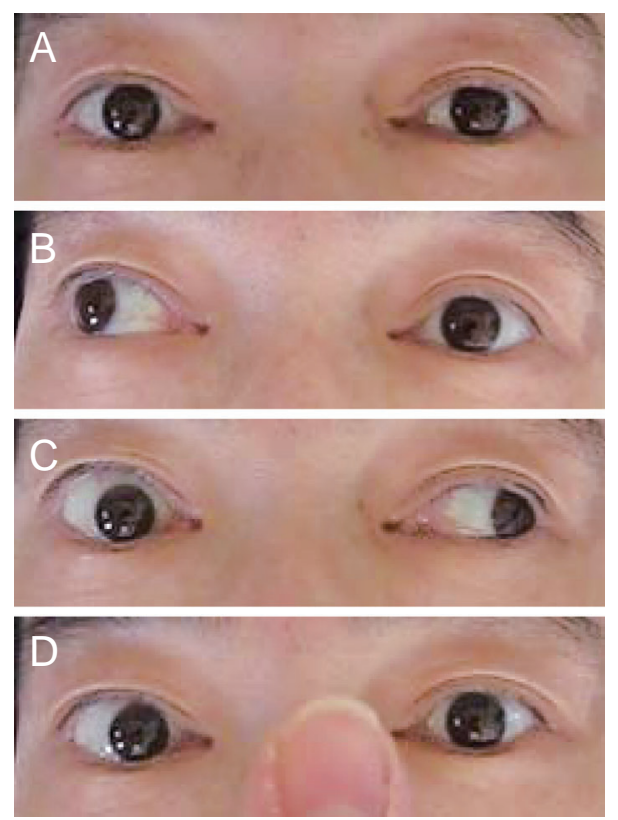

Figure 1. Photograph of eye movement: Panel (A) shows no deviation in primary gaze. Restriction of adduction in horizontal gaze was noted bilaterally $(\mathrm{B}, \mathrm{C})$. Convergence could not be evoked (D).
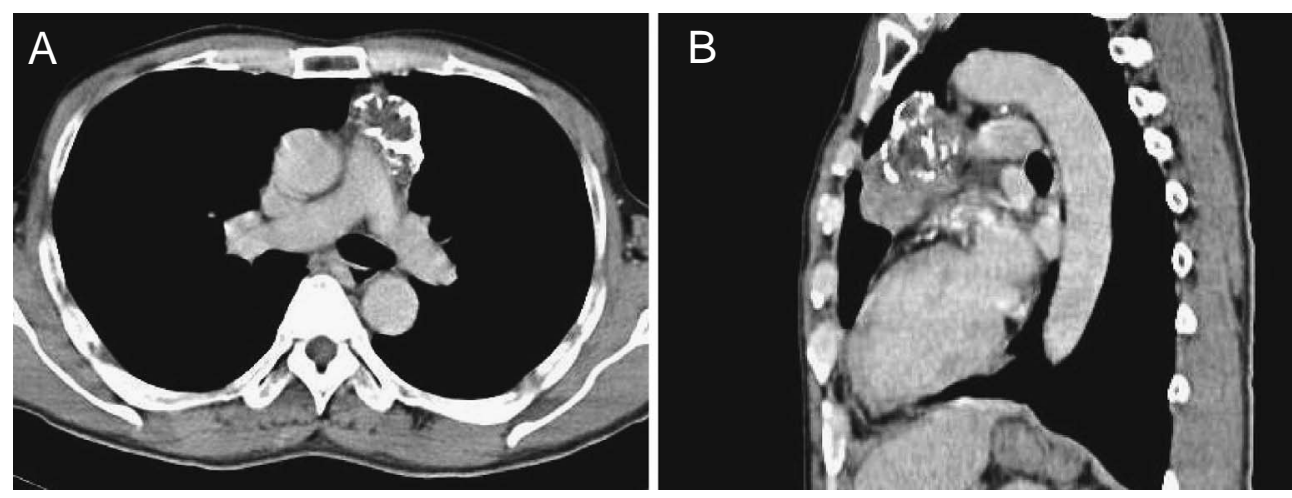

Figure 2. Chest CT revealed a $4 \mathrm{~cm}$ calcifying anterior mediastinal tumor, with heterogeneous internal density with contrast medium enhancement (A: axial section, B: sagittal section).

tory evaluation showed an elevation of serum CK of 1,570 IU/L (normal level <180 IU/L) and hypothyroidism with an increased thyroid stimulating hormone (TSH) level of 55.9 $\mathrm{mIU} / \mathrm{mL}$ (normal range, 0.4-6.0 $\mathrm{mIU} / \mathrm{mL}$ ), a decreased free T4 level of $0.7 \mathrm{ng} / \mathrm{dL}$ (normal range $0.8-1.6 \mathrm{ng} / \mathrm{dL}$ ), and a normal free T3 level of $2.6 \mathrm{pg} / \mathrm{dL}$ (normal range 1.5-3.5 pg/ dL). On further evaluation, anti-thyroperoxidase (TPO) antibody level was $6.3 \mathrm{IU} / \mathrm{mL}$ (normal level $<0.3 \mathrm{IU} / \mathrm{mL}$ ).

Although these clinical findings were attributed to ophthalmopathy and myopathy due to autoimmune thyroiditis, based on his unusual eye movement mimicking bilateral internuclear ophthalmoplegia (INO) and deteriorating clinical course, consideration was given to a myasthenia gravis (MG). Testing by administering edrophonium almost completely abolished the nystagmus and adducting muscle weakness and improved the strength of proximal muscle groups. Repetitive nerve stimulation test showed a signifi- cant decremental response at $3-\mathrm{Hz}$ stimulation in trapezius muscle. Acetylcholine receptor binding antibody titer was increased at $133.0 \mathrm{nmol} / \mathrm{L}$ (normal level $<0.3 \mathrm{nmol} / \mathrm{L}$ ). A chest CT revealed an invasive thymoma (Fig. 2). MRI of the orbits (Fig. 3) and brainstem was unremarkable. There were no plaques or white matter lesions suggestive of multiple sclerosis on MRI (Fig. 4).

Our final diagnosis was generalized MG Osserman grade III with autoimmune thyroiditis. After an extended thymectomy and thoracic radiation, his symptoms were effectively controlled with prednisolone, cholinesterase inhibitors and levothyroxine. When last seen, in December 2008, he was well.

\section{Discussion}

This patient presented with eye movement mimicking bi- 


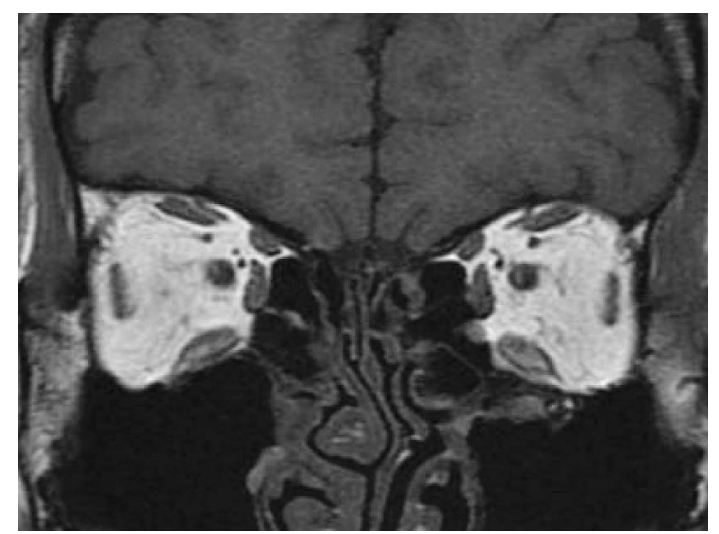

Figure 3. MRI of orbit showed no enlargement of extraocular muscles suggestive of dysthyroid ophthalmopathy (T1weighted image).

lateral internuclear ophthalmoplegia (INO). INO refers to an abnormality of conjugate horizontal eye movement that is characterized by failure of adduction in one eye and nystagmus in the abducting eye. True INO is caused by a lesion in the medial longitudinal fasciculus (MLF), commonly resulting from multiple sclerosis or stroke (3), whereas 'pseudoINO' results from peripheral conduction defects, such as those occurring in Miller Fisher syndrome and MG (3).

The history and clinical examination suggested that he was suffering from generalized MG and autoimmune thyroiditis. While both diseases can cause ophthalmopathy as well as fatigue and weakness, the positive tensilon test in which intravenous edrophonium completely abolished the nystagmus and adducting muscle weakness strongly suggested the 'pseudo-INO' of myasthenic origin. On the other hand, the fact that neither exophthalmos nor extraocular muscle enlargement was observed on orbital MRI suggested that a diagnosis of pseudo-INO caused by dysthyroid ophthalmopathy was unlikely. Furthermore, although involvement of the medial rectus muscle in dysthyroid ophthalmopathy is well established (4) 'pseudo-INO' caused by dysthyroid ophthalmopathy has not been reported to date.

The possible mechanism of 'pseudo-INO' in the present patient is as follows: For horizontal gaze, precise coordination between cranial nerves III, IV, and VI and their interneuronal pathways that project through the MLF is necessary. A lesion of the MLF can interrupt these inputs and result in a mismatch of neuronal activity that innervates the medial rectus muscle, contraction of which causes the eye to adduct. In our patient, interference with these impulses at the myoneural junction likely resulted in a similar clinical picture, because edrophonium, acting on neuromuscular transmission, abolished this abnormality (5). The fact that

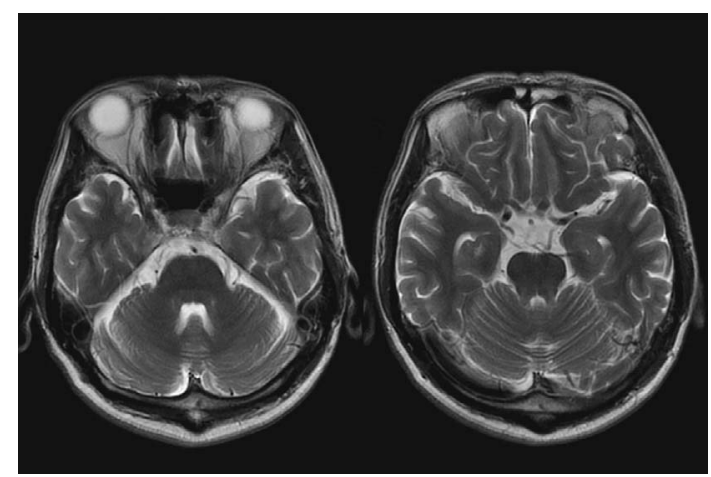

Figure 4. MRI of the brainstem showed no high intensity lesions (T2-weighted image).

the nystagmus as well as the eye movement was abolished following administration of edrophonium might suggest that the to-and-fro movement of the abducting eyes was the result of central compensation for the adduction failure (6). On the other hand, the reason why our patient showed this unique involvement pattern of ocular muscles is unclear because the ocular manifestations of MG may mimic any 'neurogenic' motility pattern (7) and anecdotal reports yield no consensus for the extraocular muscle involvement (8), although some authors reported that the medial rectus muscles are most commonly affected (9).

In contrast to our patient, previously reported MG cases of 'pseudo-INO' in adults had mild clinical expressions, with preferential ocular involvement and a lower frequency of thymic diseases and thyroid disorders (5, 6, 10-16). This may be because a detailed ocular examination, on which a diagnosis of ocular MG naturally depends, is not always essential for a diagnosis of generalized MG. Indeed, also in our patient, 'pseudo-INO' was not a critical manifestation for the diagnosis of generalized MG, but our case suggested that in a setting in which hypothyroidism and generalized MG coexist, recognition of this unique symptom may be helpful for avoiding a diagnostic pitfall for the latter.

Because a normal MRI of the brain does not completely rule out multiple sclerosis, a possibility that a co-occurrence of multiple sclerosis and myasthenia gravis manifested by INO may exist (17). Although no plaques suggestive of multiple sclerosis were observed, a careful clinical follow-up including MRI and cerebrospinal fluid examination would be necessary.

In conclusion, we report a patient with the co-occurrence of hypothyroidism and generalized $\mathrm{MG}$ as a pitfall for the latter. In similar cases, the recognition of myasthenic 'pseudo-INO' may be helpful for an earlier correct diagnosis.

\section{References}

1. Marino M, Ricciardi R, Pinchera A, et al. Mild clinical expression of myasthenia gravis associated with autoimmune thyroid diseases. J Clin Endocrinol Metab 82: 438-443, 1997.
2. Vargas ME, Warren FA, Kupersmith MJ. Exotropia as a sign of myasthenia gravis in dysthyroid ophthalmopathy. Br J Ophthalmol 77: 822-823, 1993. 
3. Keane JR. Internuclear ophthalmoplegia: unusual causes in 114 of 410 patients. Arch Neurol 62: 714-717, 2005.

4. Spoor TC, Martinez AJ, Kennerdell JS, Mark LE. Dysthyroid and myasthenic myopathy of the medial rectus: a clinical pathologic report. Neurology 30: 939-944, 1980.

5. Bakheit AM, Behan PO, Melville ID. Bilateral internuclear ophthalmoplegia as a false localizing sign. J R Soc Med 84: 627, 1991.

6. Ito K, Mizutani J, Murofushi T, Mizuno M. Bilateral pseudointernuclear ophthalmoplegia in myasthenia gravis. ORL J Otorhinolaryngol Relat Spec 59: 122-126, 1997.

7. Osher RH. Myasthenic "oculomotor" palsy. Ann Ophthalmol 11: 31-34, 1979.

8. Cleary M, Williams GJ, Metcalfe RA. The pattern of extra-ocular muscle involvement in ocular myasthenia. Strabismus 16: 11-18, 2008

9. Oosterhuis HJ. The ocular signs and symptoms of myasthenia gravis. Doc Ophthalmol 52: 363-378, 1982.

10. Glaser JS. Myasthenic psuedo-internuclear ophthalmoplegia. Arch Ophthalmol 75: 363-366, 1966.
11. Cogan DG. Internuclear ophthalmoplegia, typical and atypical. Arch Ophthalmol 84: 583-589, 1970.

12. Acers TE. Ocular myasthenia gravis mimicking pseudointernuclear ophthalmoplegia and variable esotropia. Am J Ophthalmol 88: 319-321, 1979.

13. Jay WM, Nazarian SM, Underwood DW. Pseudo-internuclear ophthalmoplegia with downshoot in myasthenia gravis. J Clin Neuroophthalmol 7: 74-76, 1987.

14. Ehongo A, Cordonnier M, Van Nechel C, et al. [Internuclear bilateral pseudo-ophthalmoplegia and dermatomyositis]. Bull Soc Belge Ophtalmol 263: 43-51, 1996 (in French).

15. Fagundez Vargas MA, Andres Domingo ML, Calvo Arrabal MA. [The WEBINO syndrome as presentation form of myasthenia gravis]. Arch Soc Esp Oftalmol 75: 485-488, 2000 (in Spanish).

16. Khanna S, Liao K, Kaminski HJ, Tomsak RL, Joshi A, Leigh RJ. Ocular myasthenia revisited: insights from pseudo-internuclear ophthalmoplegia. J Neurol 254: 1569-1574, 2007.

17. Aita JF, Snyder DH, Reichl W. Myasthenia gravis and multiple sclerosis: an unusual combination of diseases. Neurology 24: 7275,1974

(C) 2010 The Japanese Society of Internal Medicine http://www.naika.or.jp/imindex.html 